\title{
G6PD deficiency and severity of COVID19 pneumonia and acute respiratory distress syndrome: tip of the iceberg?
}

\author{
Jihad G. Youssef ${ }^{1} \cdot$ Faisal Zahiruddin ${ }^{1} \cdot$ George Youssef $^{2}$. Sriram Padmanabhan ${ }^{3}$ - Joe Ensor ${ }^{4}$ - Sai Ravi Pingali ${ }^{4}$. \\ Youli $\mathrm{Zu}^{5}$. Sandeep Sahay ${ }^{6} \cdot$ Swaminathan P. Iyer ${ }^{3,7}$
}

Received: 7 August 2020 / Accepted: 3 January 2021 / Published online: 13 January 2021

(C) The Author(s), under exclusive licence to Springer-Verlag GmbH, DE part of Springer Nature 2021

\begin{abstract}
The severe pneumonia caused by the human coronavirus (hCoV)-SARS-CoV-2 has inflicted heavy casualties, especially among the elderly and those with co-morbid illnesses irrespective of their age. The high mortality in African-Americans and males, in general, raises the concern for a possible X-linked mediated process that could affect the viral pathogenesis and the immune system. We hypothesized that G6PD, the most common X-linked enzyme deficiency, associated with redox status, may have a role in severity of pneumonia. Retrospective chart review was performed in hospitalized patients with COVID19 pneumonia needing supplemental oxygen. A total of 17 patients were evaluated: six with G6PD deficiency (G6PDd) and 11 with normal levels. The two groups (normal and G6PDd) were comparable in terms of age, sex, co-morbidities, and laboratory parameters - LDH, IL-6, CRP, and ferritin, respectively. Thirteen patients needed ventilatory support ; 8 in the normal group and 5 in the G6PDd group (72\% vs. $83 \%$ ). The main differences indicating increasing severity in normal vs. G6PDd groups included G6PD levels (12.2 vs. 5.6, $P=0.0002)$, $\mathrm{PaO} 2 / \mathrm{FiO} 2$ ratio ( 159 vs. $108, P=0.05)$, days on mechanical ventilation (10.25 vs. 21 days $P=0.04)$, hemoglobin level (10 vs. 8.1 $P=0.03$ ), and hematocrit (32 vs. $26 P=0.015$ ). Only one patient with G6PDd died; 16 were discharged home. Our clinical series ascribes a possible biological role for G6PDd in SARS-CoV2 viral proliferation. It is imperative that further studies are performed to understand the interplay between the viral and host factors in G6PDd that may lead to disparity in outcomes.
\end{abstract}

\section{Key Points}

- COVID19 studies show higher mortality in men, due to severe pneumonia and ARDS, indicating possible X-linked mediated differences

- G6PD, the most common X-linked enzymopathy, highly prevalent in African Americans and Italians, maintains redox homeostasis.

- Preclinical studies using G6PD deficient (G6PDd) cells infected with human coronavirus (hCoV), show impaired cellular responses, viral proliferation and worsening oxidative damage.

- Retrospective chart review in hospitalized patients with COVID19 pneumonia needing supplemental oxygen shows differences between the two groups (Normal and G6PDd) in hematological indices; the G6PDdgroup demonstrated prolonged PaO2/ $\mathrm{FiO} 2 \mathrm{ratio}$, and longer days on mechanical ventilation indicating the severity of the pneumonia.

Swaminathan P. Iyer spiyer@mdanderson.org

1 Division of Pulmonary and Critical Care Medicine, Houston Methodist Pulmonary Transplant Center, Houston Methodist Hospital, Houston, TX, USA

2 College of Natural Sciences and Mathematics, University of Houston, Houston, TX, USA

3 Collaborative Action for SARS-CoV-2 Eradication (CARE), Houston, TX, USA
4 Houston Methodist Cancer Center, Houston Methodist Research Institute, Houston Methodist Hospital, Houston, TX, USA

5 Houston Methodist Department of Pathology and Genomic Medicine, Houston Methodist Research Institute, Houston Methodist Hospital, Houston, TX, USA

6 Division of Pulmonary and Critical Care Medicine, Houston Methodist Lung Center, Houston Methodist Research Institute, Houston Methodist Hospital, Houston, TX, USA

7 Department of Lymphoma/Myeloma, Division of Cancer Medicine, University of Texas MD Anderson Cancer Center, 1400 Unit 429, Holcombe Blvd, Houston, TX 77030, USA 
Keywords COVID19 · G6PD · African American · X-linked

\section{Introduction}

The novel coronavirus SARS-CoV2 that causes coronavirus disease 2019 (COVID19) has approximately afflicted over 72 million people worldwide [1-4], including approximately 16.2 million in the USA as of December 2020. Among 1482 patients hospitalized reported by COVID19-Associated Hospitalization Surveillance Network (COVID-NET), $74.5 \%$ were aged $\geq 50$ years, and $54.4 \%$ were male $[2,5,6]$. The male predominance hints at $\mathrm{X}$ linked related differences in the predilection to the severity of illness. One such possibility is the X-linked glucose-6-phosphate dehydrogenase (G6PD) deficiency (G6PDd), the most common enzymopathy, commonly manifesting as hemolysis due to oxidative stress [7-9]. With over 200 mutations identified in G6PDd, mainly in the coding regions, causing various degrees of deficiency, it is found in high frequency among African Americans, Mediterranean, and Asians [8]. The gene encoding G6PD is located near the telomeric region of the distal arm of the X chromosome (band Xq28), a welldocumented hot spot of a group of genes that includes fragile $\mathrm{X}$, color vision, hemophilia $\mathrm{A}$, and congenital dyskeratosis [8]. The G6PD gene results in many biochemical variants and the deficiency of the gene product-G6PD, which is the rate-limiting enzyme in the pentose-phosphate pathway $[8,9]$. The variants are grouped into four classes: (a) Class I variants comprise the most severe form of G6PDd and lead to chronic non-spherocytic hemolytic anemia, and typically occurs with enzyme activity $<10 \%$ of normal; (b) Class II variants typically have $<10 \%$ residual enzyme activity, but no hemolytic anemia; and (c) Class III and IV variants (10-60\% and 60$150 \%$ activity, respectively) have milder phenotypes and hemolysis occurs only after extreme oxidative stress [10]. Very severe G6PDd is sporadic and rare, whereas less severe deficiencies are polymorphic, and more common in tropical areas, postulated to be evolved as protection against malaria [7, 8]. Males are more commonly affected when hemizygous and can be either phenotypically normal or deficient [10]. Homozygous females are as deficient as the hemizygous males, whereas heterozygous females are mosaics with intermediate levels of deficiency as a result of random $\mathrm{X}$ chromosome inactivation (lyonization) [11]. We report here clinical scenarios of six COVID19 positive patients, with no previous respiratory issues with G6PDd, who required longer ventilatory support and ICU care compared to 11 matched controls. Given the role of G6PD in altering redox homeostasis [12], we hypothesize that the deficiency further enhances oxidative stress by uncontrolled production of reactive oxygen species (ROS) during this rapidly evolving inflammation caused by the SARS-CoV-2 virus and host immune system, leading to abnormal pulmonary vascular performance and respiratory decline. This case series warrants a systematic investigation of the role played by G6PD in this unrelenting COVID19-induced pneumonitis.

\section{Methods}

We reviewed the charts of 17 consecutive patients who were admitted to the Houston Methodist hospital and ICU with confirmed COVID19 infection, and where G6PD levels were available with consent, between March 15 and May 15, 2020. Clinical data were obtained through an Institutional Review Board (IRB) approved protocol PRO00025607 that allowed review of medical records. The main inclusion criteria included adults $>18$ years old with a diagnosis of COVID19 within $24 \mathrm{~h}$ of admission to the hospital and where G6PD levels were obtained. G6PD was considered low if the recorded values were below the laboratory cut-off value of $9.6 \mathrm{U} / \mathrm{g} \mathrm{Hb} ;<4.5$ was considered severe, values between 4.5 and 9.6 as mild, and all other values as normal. Of note, G6PD testing as obtained as baseline in patients as hydroxychloroquine (HCQ) was the first line of treatment per the institutional protocol at the time of the study. Both groups received a 5-day course of HCQ and completed the course by the time G6PD result was reported. The G6PDd patients (3 severe and 3 mild) were compared to the normal cohorts (where the G6PD values were $>9.6$ ) in 2:1 matching for age, gender, and co-morbidity. The data reported here have follow-up available through June 20, 2020; each patient had at least 21 days of follow-up.

\section{Statistical analysis}

Descriptive statistics were used to summarize the data; results are reported as means and medians, as appropriate. The unpaired $t$ test was performed using GraphPad Prism 8 for macOS version 8.4.2 (464), April 7, 2020, GraphPad Software, San Diego California USA, www.graphpad.com. All comparisons were one-tailed; a $P$ value of less than 0.05 was considered significant.

\section{Results}

The reverse-transcriptase-polymerase-chain-reaction (RTPCR) for SARS-CoV2 from samples obtained from the nasopharyngeal swab was positive in 17 patients -6 with G6PDd and 11 with normal G6PD levels at the time of hospitalization. We used the two groups (Normal and G6PDd) for comparison as they were matched by age-median of 53.3 vs. 53.5 years $(P$ 
value of 0.49 ), gender ( $50 \%$ men), and similar co-morbidities such as diabetes, hypertension, and obesity with body mass index (BMI) of 35.5 vs.31.2 ( $P$ value 0.39 ), respectively. The G6PDd cohort had a higher number of African-Americans (66\% vs, $45 \%$ ) than the normal cohort (Table 1). All patients presented with cough and shortness of breath. The chest computed tomography (CT) at admission showed variations of bilateral peripheral patchy ground-glass opacities but highly suggestive of COVID19 pneumonia. All patients received standard of care treatment as approved by the hospital COVID19 task force, and this included supportive care, HCQ, and in some instances compassionate use remdesivir, convalescent plasma, and anticoagulation if clinically not contraindicated.

No patient tested positive for other respiratory viruses or had any other documented bacterial infections. Thirteen out of 17 patients needed ventilatory support with 8 in the normal group and 5 in the G6PDd group. Laboratory data (Table 2) were significant for elevated lactate dehydrogenase (LDH), Creactive protein (CRP), interleukin-6 (IL-6), and ferritin, with no statistical differences between the two groups. The significant difference between the normal and G6PDd patients
(Fig. 1a-g) was noticed for G6PD levels (12.2 vs. 5.6, $P=0.0002)$, partial pressure of oxygen/fraction of inspired oxygen $(\mathrm{PaO} 2 / \mathrm{FiO} 2)$ ratio (159 vs. $108, P=0.05)$, days on mechanical ventilation ( 10.25 vs. 21 days $P=0.04)$, hemoglobin level (10 vs. $8.1 P=0.03$ ), and hematocrit (32 vs. 26 $P=0.01)$. Three patients required blood transfusion ( 1 in normal and 2 in the G6PDd groups, respectively). Only one patient with G6PDd died as the family decided to withdraw care. Of the 16 surviving patients, five were discharged to rehabilitation units, and 11 were discharged from the ICU to the general wards and eventually home (Fig. 1g).

\section{Discussion}

The emerging data from COVID19 shows that the case fatality rate (CFR) is higher in males, indicating a possible $\mathrm{X}$ linked mediated difference in the crosstalk between the SARS-CoV-2 and immune effectors [2, 5, 6]. We illustrate the potential role of G6PDd in patients hospitalized for COVID19 pneumonia affecting the severity of the disease and the clinical management. Thus far, there are no reports of an association between G6PDd and COVID19, likely

Table 1 Patient characteristics

\begin{tabular}{|c|c|c|c|}
\hline Characteristics & Normal G6PD $(n=11)$ & Deficient G6PD $(n=6)$ & $\begin{array}{l}\text { One tailed } t \text { test } \\
(P \text { value })\end{array}$ \\
\hline Age (median) & 53.3 & 53.5 & 0.49 \\
\hline \multicolumn{4}{|l|}{ Sex: } \\
\hline Female & 5 & 3 & \multirow[t]{2}{*}{0.63} \\
\hline Male & 6 & 3 & \\
\hline \multicolumn{4}{|l|}{ Race/ethnicity } \\
\hline African American & 5 & 4 & \multirow[t]{5}{*}{0.38} \\
\hline Caucasian & 5 & 0 & \\
\hline Hispanic & 0 & 1 & \\
\hline Asian & 0 & 1 & \\
\hline Prefer not to say & 1 & 0 & \\
\hline Body mass index (median) & 35.5 & 31.2 & 0.39 \\
\hline \multicolumn{4}{|l|}{ Co-morbidities } \\
\hline Hypertension & 2 & 1 & \\
\hline Diabetes mellitus & 4 & 1 & \\
\hline $\mathrm{BMI}>36$ & 3 & 2 & \\
\hline Previous malignancy & 1 & 0 & \\
\hline G6PD mean, U/g Hb & $12.2(9.6-15.3)$ & $5.6(1.8-9.3)$ & 0.0002 \\
\hline Symptoms & \multicolumn{3}{|c|}{ Cough, shortness of breath, chest discomfort } \\
\hline Lowest $\mathrm{PaO} 2 / \mathrm{FiO} 2$ & 159 & 108 & 0.05 \\
\hline Mean Rothman Index & 58.63 & 68.8 & 0.12 \\
\hline Mean Length of stay (days) & $18(7-32)$ & $23.5(7-51)$ & 0.18 \\
\hline Wait and watch days before intubation & $2.5(1-6)$ & $4.8(3-7)$ & 0.06 \\
\hline Length of mechanical ventilation (days) & $10.25(4-16)$ & $21(5-46)$ & 0.04 \\
\hline \multicolumn{4}{|l|}{ Discharge status } \\
\hline Home & 10 & 2 & - \\
\hline Rehabilitation/long-term facility & 3 & 2 & \\
\hline
\end{tabular}


Table 2 Laboratory characteristics

\begin{tabular}{llll}
\hline Characteristics & $\begin{array}{l}\text { Normal G6PD } \\
(n=11)(\text { range })\end{array}$ & $\begin{array}{l}\text { Deficient G6PD } \\
(n=6)(\text { range })\end{array}$ & $\begin{array}{l}\text { One tailed } t \text { test } \\
(P \text { value })\end{array}$ \\
\hline Lowest WBC, $\times 10[9] / \mathrm{L}$ & $5.2(2.1-6.9)$ & $5.1(3.0-8.3)$ & 0.16 \\
Lowest Neutrophils \% & $60(35-74)$ & $62(47-82)$ & 0.37 \\
Lowest ALC, $\times 10[9] / \mathrm{L}$ & $618(191-1917)$ & $393(111-738)$ & 0.16 \\
Lowest hemoglobin, g/dl & $10(6.5-13)$ & $8.1(6.6-11)$ & $\mathbf{0 . 0 2}$ \\
Lowest hematocrit \% of red blood cells & $32(20-40)$ & $26(22-34)$ & $\mathbf{0 . 0 1 5}$ \\
Platelets, $\times 10$ [9]/L & $425(337-626)$ & $405(179-571)$ & 0.38 \\
Peak lactate, mmol/L & $2.1(1.2-3.5)$ & $5.0(1.3-16)$ & 0.05 \\
Peak IL-6, pg/L & $577(5-2957)$ & $278(57-669)$ & 0.24 \\
Peak CRP, mg/L & $21(0.87-41)$ & $28(15-51)$ & 0.11 \\
Peak ferritin, mg/L & $1371(476-3648)$ & $7095(916-32,659)$ & 0.07 \\
Peak LDH, IU/L & $518(208-862)$ & $663(378-996)$ & 0.11 \\
Peak D-dimer & $5.7(0.41-20)$ & $13(0.6-20)$ & 0.05 \\
Peak AST, IU/L & $134(32-350)$ & $176(18-302)$ & 0.20 \\
Peak ALT, IU/L & $148(22-527)$ & $216((35-503)$ & 0.22 \\
Peak alkaline phosphatase, IU/L & $167(65-449)$ & $131(58-279)$ & 0.26 \\
Peak Total bilirubin, $\mu \mathrm{mol} / \mathrm{L}$ & $1.6(0.5-3.8)$ & $2.0(0.5-2.3)$ & 0.22 \\
Highest creatinine, $\mu \mathrm{mmol} / \mathrm{L}$ & $3.3(0.7-17)$ & $2.0(0.86-6.4)$ & 0.27 \\
Highest glucose, mg/dl & $209(91-310)$ & $242(133-378)$ & 0.23 \\
Highest triglyceride, mg/dl & $193(85-543)$ & $218(65-416)$ & 0.35 \\
Peak troponin-I, ng/ml & $0.85(0.006-6.8)$ & $0.28(0.006-0.5)$ & 0.26 \\
Highest QTc on EKG, ms & $476(437-551)$ & $486(446-528)$ & 0.32 \\
\hline & & & \\
\hline
\end{tabular}

Abbreviations: $A L T$ alanine aminotransferase, $A L T$ absolute lymphocyte count, $A S T$ aspartate aminotransferase, $I L-6$ interleukin-6, $C R P C$-reactive protein, $L D H$ lactate dehydrogenase because G6PDd was either rare or of mild variety or not reported in the respective populations [2-4]. Nevertheless, given the high prevalence of this mutation in African-Americans (1 in 10) and the Italians [5, 7], it is important to elucidate the biological differences in the outcomes to COVID19 infection. In our study, a total of 17 patients were enrolled where the G6PD levels were known - six were identified with G6PDd; we used the 11 patients with normal levels as the comparator group. In the deficiency group, two males (both AfricanAmericans) had levels less than 4.5, and the other four patients, one male and the three females (likely hemizygous), had levels just below the lower limit of the established reference $(9.6$ - $16.6 \mathrm{U} / \mathrm{g} \mathrm{Hb})$ [13].

G6PD converts glucose-6-phosphate into 6phosphogluconolactone and catalyzes the generation of reduced nicotinamide adenine dinucleotide phosphate (NADPH). Furthermore, NADPH is the critical co-factor for the enzyme glutathione reductase, which reduces glutathione disulfide (GSSG) into reduced glutathione (GSH). GSH eliminates ROS by scavenging the hydroxyl radicals, singlet oxygen, and electrophiles. The deficiency is most pronounced on erythrocytes, which depend solely on the cytosolic pentose phosphate pathway and generation of GSH for oxidative protection. Generally, the outcomes for G6PDd patients with hemolysis are favorable, if they can avoid oxidative triggers commonly encountered due to drugs (including some antimalarial drugs, sulfonamides, and rasburicase) [7, 9]. Fatigue is the most common symptom followed by dyspnea, dizziness, headache, pallor, chest pain, and jaundice if hemolysis is severe. A quantitative analysis of G6PD activity [13] can provide a definitive diagnosis of G6PD deficiency so that the individuals can be advised to avoid drugs, foods, or other oxidizing agents that may precipitate a hemolytic crisis. The severity of the hemolysis is variable and depends on the G6PD allelic mutation and factors such as the dose of the inciting drug. The usual management is supportive care and blood transfusion if hemolysis is severe.

The reason for testing G6PD in our patients was proactive baseline measurement in preparation for hydroxychloroquine use. As expected, in our study, there were significant differences in the G6PD levels that also correlated with the differences in Hemoglobin and hematocrit (Table 1). More importantly, there is increase in the severity of the pulmonary process as demonstrated by the higher requirements of oxygen and longer time on mechanical ventilation. The differences seen as a measure of lowest $\mathrm{PaO} 2 / \mathrm{FiO} 2$ is also shown by the similarity of Rothman index between admission and the time of mechanical ventilation with subsequent deterioration in the deficiency group (Table 2 and Fig. 1a-g). The limitation of this study is the retrospective review, the sample size, and the 
Fig. 1 A-G-Differences between the normal and G6PDd patients A-F- A. Differences in G6PD mean, B. Lowest Hemoglobin, C. Lowest Hematocrit, D. Lowest $\mathrm{PaO} 2 / \mathrm{FiO} 2$, E. Wait and watch days before intubation, F. Length of mechanical ventilation (LOMV) and G. Kaplan-Meier Survival Curve. A $p$ value of less than 0.05 was considered significant
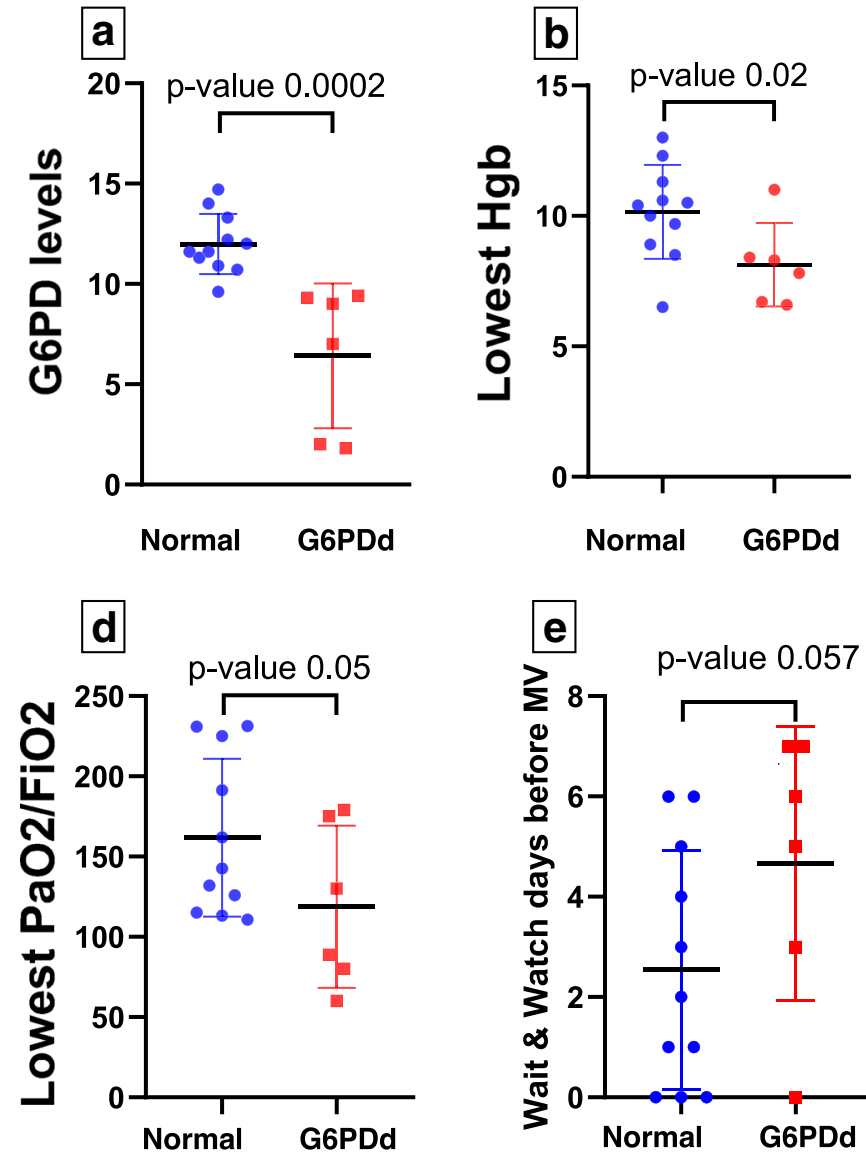
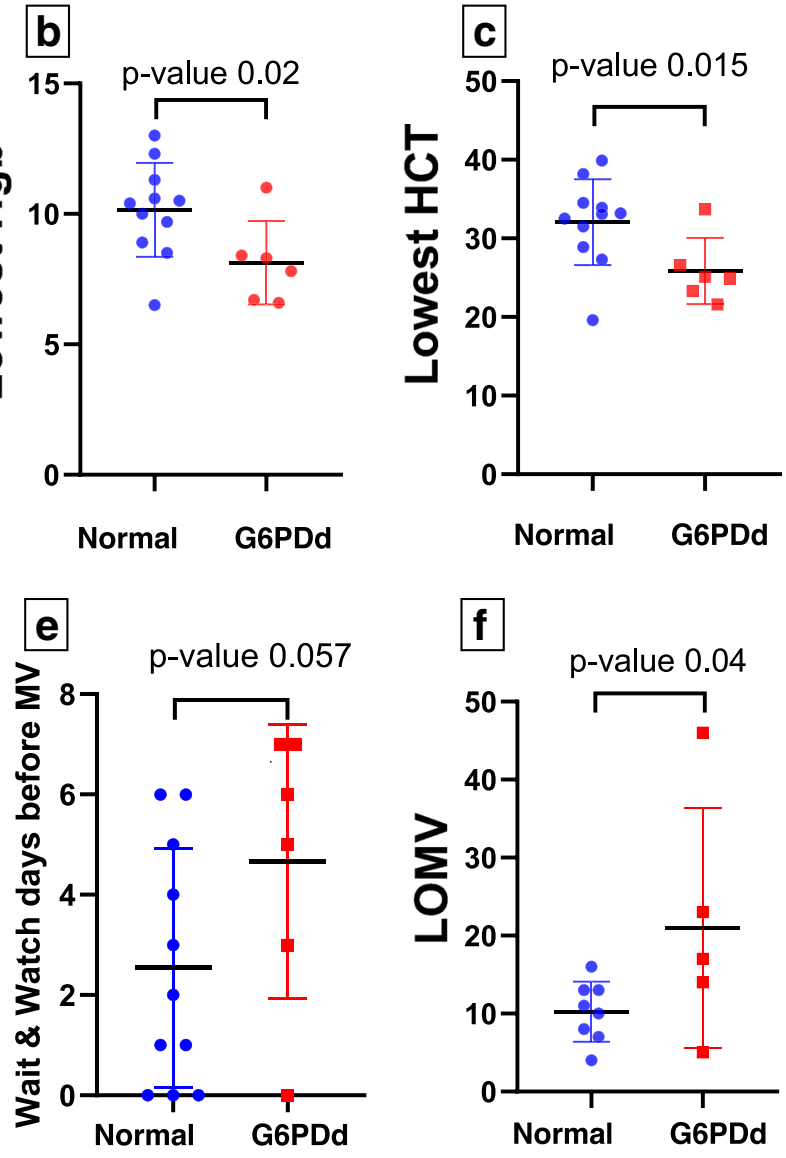

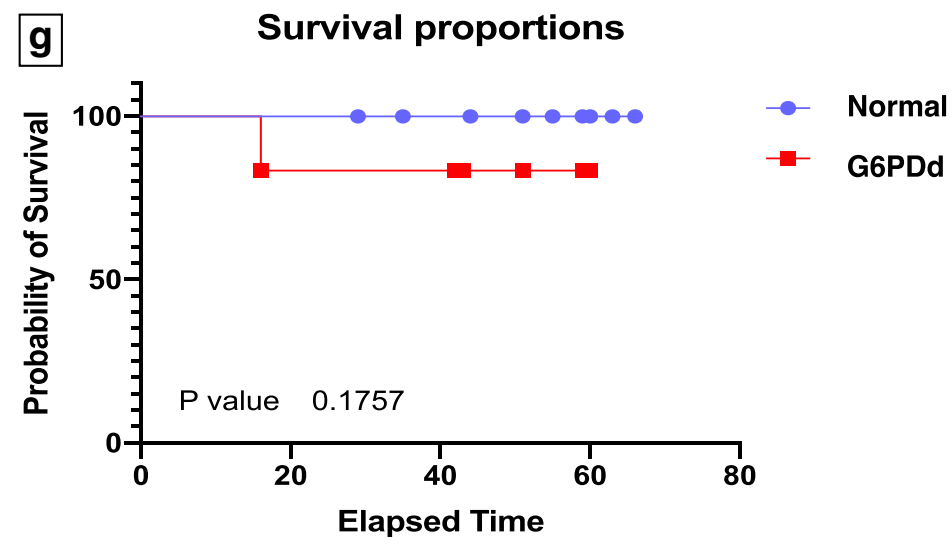

lack of power to detect the differences due to intervention. Nevertheless, given the high prevalence of this mutation in African-Americans (1 in 10) and Italians [3, 5], it is important to elucidate the biological differences in the outcomes to COVID19 infection.

Two questions that are of utmost clinical importance that need answers are as follows: (1) Does the deficiency increase the susceptibility and or the severity to COVID19? (2) How do we judiciously use the pharmacologic array of medications that can potentially worsen the deficiency? One of the severe manifestations of COVID19 is the ARDS characterized by the acute onset of hypoxemia, reduced lung compliance, diffuse lung inflammation, and bilateral opacities on chest imaging attributable to noncardiogenic pulmonary edema [14]. As details of the pathobiology are still emerging, our understanding of similar viral-mediated lung injury from the previous human coronavirus (hCoV) epidemics of Severe Acute Respiratory Syndrome (SARS) and Middle Eastern Respiratory Syndrome (MERS) points to the central oxidative stress on the pulmonary vasculature by ROS production causing further alveolar damage limiting gas exchange and setting up right to left shunting of venous blood [15-17]. The pathophysiologic 
process of pulmonary vascular endothelial diathesis is caused by increased oxidant stress and reduced bioavailable nitric acid [15-17]. This has also been reported in influenza infection, which provokes a pro-oxidant condition by increasing ROS production in the host cell to facilitate viral proliferation $[18,19]$. The ROS further inactivates nitric oxide resulting in nitric oxide insufficiency [20]. The primary determinant of protection against oxidative stress is the ability to maintain GSH stores through the synthesis of NADPH. Since NADPH concentrations are primarily maintained by G6PD encoded by its gene located on Xq28, it follows that its deficiency could result in an inability to eliminate ROS as seen in our patients.

There is now accumulating evidence that G6PD deficiency affects cells other than those of erythrocytes [12, 21-24]. The replication and spread of respiratory viruses normally involves activation of the antiviral innate immune responses and culminates in the production of type I interferons (IFNs) and proinflammatory cytokines such as tumor necrosis factoralpha (TNF- $\alpha)$ which in turn upregulate antiviral proteins [24]. In an in vitro study, when G6PD-deficient human fibroblasts and human lung epithelial carcinoma A549 cells were treated with G6PD-RNA interference (RNAi), they showed reduced viability and a threefold increase in viral replication [21]. In another study, authors report marked reduction in antiviral genes such as TNF- $\alpha$ in G6PD deficient A549 cells after infection with HCoV-229E, when compared to the parental cell lines [23]. Their observations also include decreased nuclear factor kappa-light-chain-enhancer of activated $\mathrm{B}$ cells $(\mathrm{NF}-\mathrm{KB})$ activation in virus-infected host cells, pointing to reduced ability to activate timely antiviral responses. Furthermore, a few studies indicate that oxidative stress increases the susceptibility of non-erythroid G6PDd cells to viral infection which could be ameliorated by antioxidant agents, such as lipoic acid [21].

In another study, G6PDd peripheral blood mononuclear cells (PBMCs) from patients and human monocytic (THP-1) cells showed impaired inflammasome activation. In particular, G6PD knockdown reduced the expression of mature interleukin (IL)-1 $\beta$, but not the expression of caspase-1 or the components of the inflammasome (NLRP3, ASC, and pro-caspase-1) pathway [12]. Additionally, there was a differential expression of cytokines between the G6PDd cells and the normal cells. Furthermore, as reported in severe influenza pneumonia [17, 19], this pro-oxidant condition can induce secretion of inflammatory cytokines, including interleukin (IL)-1 $\beta$, IL-6, IFN, and TNF - $\alpha$ from the microenvironment. This uncontrolled pro-inflammatory response, referred to as the cytokine storm, is abetted by the action of monocytes/ macrophages, neutrophils on the infected lung epithelial cells [22]. Taken together, these results indicate that G6PD deficiency can allow viral proliferation even as it impairs the cellular immune response through abnormal redox homeostasis.
It is imperative that further studies are performed to have an enhanced understanding of the interplay between the viral and host factors in G6PD-deficiency that may lead to disparity in outcomes. This will have significant clinical implications in the management of patients with COVID19 infection.

Acknowledgments To Marianne Younes, MA, for editorial support and to Collaborative Action for SARS-CoV-2 Eradication (CARE) Inc- a 501(c) Not for profit group.

Authors' contributions Conception and design: JGY, FZ, SS, and SPI. Manuscript writing: JGY, FZ, GY, MY, SP, JE, SP, YZ, SS and SPI. Data Collection and analysis: JGY, FZ, GY, MY, SP, JE, YZ, SS and SPI.

Final approval of manuscript: JGY, FZ, GY, MY, SP, JE, SP, YZ, SS and SPI.

Accountable for all aspects of the work: JGY, FZ, MY, SP, JE, SP, YZ, SS and SPI.

\section{Compliance with ethical standards}

Conflict of interest None for all others except for SS and SPI not pertaining to this work-SS: Consultant and advisor for United Therapeutics, Actelion, and Bayer pharmaceuticals. Also served as speaker for Liquidia technologies. SI: Research Grant and Consultant Seattle Genetics, Rhizen, Daiichi Sankyo, Trillium; Research GrantMerck, Affimed, Spectrum.

Statement of consent Clinical data were obtained through an Institutional Review Board (IRB) approved protocol PRO00025607 that allowed review of medical records.

\section{References}

1. JohnsHopkins. COVID-19 Map. @JohnsHopkins. https:// coronavirus.jhu.edu/map.html

2. Wang D, Hu B, Hu C et al (2020) Clinical characteristics of 138 hospitalized patients with 2019 novel coronavirus-infected pneumonia in Wuhan, China. Jama. https://doi.org/10.1001/jama.2020. 1585

3. Onder G, Rezza G, Brusaferro S (2020) Case-fatality rate and characteristics of patients dying in relation to COVID-19 in Italy. JAMA 2020. https://doi.org/10.1001/jama.2020.4683

4. Guan WJ, Ni ZY, Hu Y et al (2020) Clinical characteristics of coronavirus disease 2019 in China. N Engl J Med. https://doi.org/ 10.1056/NEJMoa2002032

5. Garg S KL, Whitaker M, et al. Hospitalization rates and characteristics of patients hospitalized with laboratory-confirmed coronavirus disease 2019 - COVID-NET, 14 States, March 1-30, 2020 MMWR. MMWR Morb Mortal Wkly Rep. 2020-04-15T06:45: 39Z 2020;69:458-464. doi:. https://doi.org/10.15585/mmwr. mm6915e3external icon

6. Grasselli G, Zangrillo A, Zanella A et al (2020) Baseline Characteristics and outcomes of 1591 patients infected with SARS-CoV-2 admitted to ICUs of the Lombardy Region, Italy. Jama. https://doi.org/10.1001/jama.2020.5394

7. Cappellini MD, Fiorelli G (2008) Glucose-6-phosphate dehydrogenase deficiency. Lancet 371(9606):64-74. https://doi.org/10.1016/ s0140-6736(08)60073-2

8. Gómez-Manzo S, Marcial-Quino J, Vanoye-Carlo A et al (2016) Glucose-6-phosphate dehydrogenase: update and analysis of new 
mutations around the world. International journal of molecular sciences 17(12). https://doi.org/10.3390/ijms17122069

9. Beutler E (1991) Glucose-6-phosphate dehydrogenase deficiency. N Engl J Med 324(3). https://doi.org/10.1056/ NEJM199101173240306

10. Online Mendelian Inheritance in Man, (OMIM) (2020) * 305900 GLUCOSE-6-PHOSPHATE DEHYDROGENASE; G6PD. OMIM: https://www.omim.org/entry/305900

11. Plenge R, Hendrich B, Schwartz C et al (1997) A promoter mutation in the XIST gene in two unrelated families with skewed Xchromosome inactivation. Nat Genet 17(3). https://doi.org/10.1038/ ng1197-353

12. Yen WC, Wu YH, Wu CC, Lin HR, Stern A, Chen SH, Shu JC, Tsun-Yee Chiu D (2020) Impaired inflammasome activation and bacterial clearance in G6PD deficiency due to defective NOX/p38 MAPK/AP-1 redox signaling. Redox Biol 28:101363. https://doi. org/10.1016/j.redox.2019.101363

13. Powers JLHBD, Grenache DG (2020) Genotype-phenotype correlations of glucose-6-phosphate-deficient variants throughout an activity distribution. J Appl Lab Med 2(6):841-850. https://doi.org/ 10.1373/jalm.2017.024935

14. Wu C, Chen X, Cai Y et al (2020) Risk factors associated with acute respiratory distress syndrome and death in patients with coronavirus disease 2019 pneumonia in Wuhan, China. JAMA Intern Med. https://doi.org/10.1001/jamainternmed.2020.0994

15. Liu J, Zheng X, Tong Q, Li W, Wang B, Sutter K, Trilling M, Lu M, Dittmer U, Yang D (May 2020) Overlapping and discrete aspects of the pathology and pathogenesis of the emerging human pathogenic coronaviruses SARS-CoV, MERS-CoV, and 2019nCoV. J Med Virol 92(5):491-494. https://doi.org/10.1002/jmv. 25709

16. Winterbourn CC (May 2008) Reconciling the chemistry and biology of reactive oxygen species. Nat Chem Biol 4(5):278-286. https://doi.org/10.1038/nchembio.85
17. Khomich OA, Kochetkov SN, Bartosch B, Ivanov AV (2018) Redox biology of respiratory viral infections. Viruses 10(8). https://doi.org/10.3390/v10080392

18. Teijaro J, Walsh K, Cahalan S et al (2011) Endothelial cells are central orchestrators of cytokine amplification during influenza virus infection. Cell. 146(6). https://doi.org/10.1016/j.cell.2011.08. 015

19. Herold S, Becker C, Ridge KM, Budinger GR (May 2015) Influenza virus-induced lung injury: pathogenesis and implications for treatment. Eur Respir J 45(5):1463-1478. https://doi.org/10. 1183/09031936.00186214

20. Short K, Kroeze E, Fouchier R, Kuiken T (2014) Pathogenesis of influenza-induced acute respiratory distress syndrome. Lancet Infect Dis 14(1). https://doi.org/10.1016/S1473-3099(13)70286-X

21. Wu YH, Tseng CP, Cheng ML, Ho HY, Shih SR, Chiu DT (2008) Glucose-6-phosphate dehydrogenase deficiency enhances human coronavirus 229E infection. J Infect Dis 197(6):812-816. https:// doi.org/10.1086/528377

22. Channappanavar R, Perlman S (Jul 2017) Pathogenic human coronavirus infections: causes and consequences of cytokine storm and immunopathology. Semin Immunopathol 39(5):529-539. https:// doi.org/10.1007/s00281-017-0629-x

23. Wu YH, Chiu DT, Lin HR, Tang HY, Cheng ML, Ho HY (2015) Glucose-6-phosphate dehydrogenase enhances antiviral response through downregulation of NADPH sensor HSCARG and upregulation of NF-kappaB signaling. Viruses 7(12):6689-6706. https:// doi.org/10.3390/v7122966

24. Channappanavar R, Zhao J, Perlman S (Aug 2014) T cell-mediated immune response to respiratory coronaviruses. Immunol Res 59(13):118-128. https://doi.org/10.1007/s12026-014-8534-z

Publisher's note Springer Nature remains neutral with regard to jurisdictional claims in published maps and institutional affiliations. 